\title{
Eficácia da Toxina Botulínica no Tratamento da Hiperidrose
}

\author{
Lislane Dias* \\ Lorena Marçal* \\ Maíra Rodrigues* \\ Túlio C. A. Alves** \\ Milena P. Pondé***
}

\begin{abstract}
RESUM 0
A hiperidrose é caracterizada por um suor excessivo e sem controle decorrente de uma hiperatividade simpática. Essa afecção pode causar sérios problemas sociais, psicológicos e ocupacionais. Os tratamentos convencionais como o uso de antiperspirantes, iontoforese ou a simpatectomia não são eficazes em casos mais severos, ou então são muito arriscados. A toxina botulínica tipo A surgiu como um tratamento seguro e eficaz da hiperidrose, ao bloquear a liberação da acetilcolina nas membranas pré-sinápticas. Este estudo tem como objetivo revisar criticamente a literatura sobre o tema. Os resultados apresentados foram obtidos de seis trabalhos realizados com pacientes que comprovadamente tinham hiperidrose. A redução percentual da sudorese foi de $86 \%$ no estudo realizado por Heckmann et al. (2001), 69,1\% no de Kinkelin et al., 63,7\% no estudo de Heckmann et al. (1999) e de 69\% nos de Solomon e Itayman. Poucos efeitos colaterais foram relatados, sendo eles dor e ardência durante as injeções e fraquezas transitórias dos músculos do polegar e da testa. O tratamento da hiperidrose palmoplantar, axilar e frontal com a toxina botulínica tem se mostrado eficaz e seguro, reabilitando o indivíduo às suas atividades no mesmo dia. A única desvantagem desse tratamento é o seu alto custo, que limita significativamente o seu uso.
\end{abstract}

Unitermos: Hiperidrose, tratamento, toxina botulínica.

\section{Introdução}

O suor é necessário para regulação da temperatura corpórea, sendo a sua produção controlada pelo sistema nervoso autônomo simpático. A hiperatividade das glândulas sudoríparas écrinas leva à transpiração excessiva, principalmente nas regiões plantar, axilar e palmar. Essa condição é conhecida como hiperidrose $^{1}$. Trata-se de uma afecção benigna, com incidência relatada entre $0,6 \%$ a $1 \%$ da população mundial (hiperidrose primária). Pode ser de origem primária - sem causa conhecida - ou secundária, associada à obesidade, menopausa, drogas antidepressivas e álcool ${ }^{2}$. A hiperidrose é uma situação desconfortável, constrangedora, acarretando prejuízos sociais, profissionais e psíquicos aos seus portadores. Em casos mais graves, ocorre gotejamento espontâneo na região afetada, a pele pode ficar macerada ou mesmo fissurada. O odor fétido pode surgir acompanhado ou não de infecções piogênicas, fúngicas e dermatites de contato ${ }^{1}$.

As formas de tratamento da hiperidrose podem ser agrupadas em paliativas e definitivas. Os antiperspirantes (cloreto de alumínio a 20\%) têm efeitos pouco duradouros - em média sete dias - além de causar irritação e tolerância. O uso de iontoforese só impede a sudorese durante o tratamento, sendo sua eficácia limitada ${ }^{3}$. As drogas anticolinérgicas, também paliativas, causam efeitos colaterais como alterações da visão, boca seca, sedação e náusea ${ }^{1}$. A remoção das glândulas axilares por excisão ou lipossucção é uma terapia definitiva, mas pode provocar hemorragia, infecção, parestesia, cicatrizes e reinervação. Outra forma definitiva é a simpatectomia, que consiste na secção do tronco simpático localizado no interior da

\footnotetext{
* Acadêmicas da Escola Baiana de Medicina e Saúde Pública (EBMSP).

** Professor Titular de Farmacologia da Escola Baiana de Medicina e Saúde Pública.

*** Professora-adjunta de Farmacologia da Escola Baiana de Medicina e Saúde Pública.
} 
cavidade torácica. Apesar de eficaz, essa técnica associa-se a risco de pneumotórax, hemotórax e síndrome de Horner ${ }^{3}$.

Na última década, a introdução da toxina botulínica tipo A no tratamento da hiperidrose tem se mostrado bastante eficaz ${ }^{4}$. Essa toxina é produzida pela bactéria anaeróbia Clostridium botulinum, que também dá origem a outros seis sorotipos (B, C, D, E, F e G). O tipo A é o mais conhecido e estudado dentre esses 5 . A toxina botulínica tipo A aglutina-se nas terminações nervosas das fibras simpáticas pós-ganglionares que inervam as glândulas sudoríparas ${ }^{4}$. Ela então é internalizada via endocitose e depois liberada no citoplasma axonal. Ao seccionar a proteína de membrana celular SNAP-25, necessária para liberação da acetilcolina, a toxina impede a liberação desse neurotransmissor ${ }^{6}$. As glândulas passam, portanto, a não receber o estímulo para a secreção. Com base nesse mecanismo de ação e nos últimos achados sobre o uso clínico da toxina botulínica, este estudo propõe revisar criticamente a literatura sobre a eficácia da toxina botulínica tipo A no tratamento da hiperidrose.

\section{Material e métodos}

Este trabalho revisa a literatura sobre a aplicação da toxina botulínica na hiperidrose. Utilizou-se como base de dados o Medline e Mdconsult. As palavraschave usadas foram Hyperhidrosis e Botulinum toxin. Foram encontrados um total de oito trabalhos. Devido à escassez de artigos científicos publicados na literatura abordando essa temática, todos os trabalhos foram incluídos, apesar de não serem estudos randomizados e duplo-cegos, e conterem, na sua maioria, um número reduzido de pacientes.

\section{Resultados}

Os resultados apresentados pelos seis trabalhos indicam uma ação satisfatória da toxina botulínica tipo A na diminuição da sudorese. No estudo de Heckmann et al. ${ }^{6}, 145$ voluntários que produziam cerca de $490 \mathrm{~g}$ de suor em cada axila foram tratados com injeções da toxina cujas dosagens não foram relatadas pelo trabalho. Duas semanas depois, houve uma redução da taxa diária de suor para $67 \mathrm{~g}$. Efeitos colaterais não foram citados. Karamfilov et $a l .^{7}$ aplicaram a toxina em 24 pacientes com faixa etária entre 19 e 58 anos com hiperidrose axilar. A dose usada foi de $200 \mathrm{U}$ por axila. Após seis dias, todos os pacientes tiveram cessação do suor excessivo. Seus efeitos duraram em média dez meses, e em quatro pacientes duraram sete meses. Dor temporária e ardor durante as injeções foram os únicos efeitos colaterais relatados.

Dez homens com hiperidrose frontal foram tratados com doses de $86 \mathrm{UM}$ de toxina por Kinkelin et al. ${ }^{8}$. Obteve-se uma redução de $173,8 \mathrm{mg} / \mathrm{min}$ de suor para $53,7 \mathrm{mg} / \mathrm{min}$, medida pelo teste da gravimetria, após quatro semanas da aplicação. Os efeitos duraram cerca de cinco meses em nove dos dez pacientes. Os efeitos adversos incluíam ardência durante a injeção e fraqueza transitória dos músculos da testa. Heckmann et al. ${ }^{3}$ realizaram um estudo com 12 pacientes afetados pela hiperidrose axilar. A dose utilizada por axila foi de $250 \mathrm{U}$. Antes do tratamento, o grau de sudorese entre eles variava de $150 \mathrm{mg} / \mathrm{min}$ a $890 \mathrm{mg} / \mathrm{min}$, medido pelo teste da gravimetria. Sete dias após, esse valor caiu para $50 \mathrm{mg} / \mathrm{min}$ em todos os pacientes. Quatro deles sentiram apenas uma dor aguda durante o primeiro dia depois da aplicação. Em sete pacientes a anidrose durou doze meses, tendo

Tabela 1 Resultado dos estudos

\begin{tabular}{|c|c|c|c|c|c|c|}
\hline Autor/ano & $\mathrm{N}^{\circ}$ de pacientes & Local & Dose & $\begin{array}{l}\text { Duração } \\
\text { média da } \\
\text { anidrose }\end{array}$ & $\begin{array}{c}\text { Efeitos } \\
\text { adversos }\end{array}$ & \% da redução \\
\hline Heckmann et al., 2001 & 145 & Axila & Não relatado & 6 meses & Não relatado & $86 \%$ \\
\hline Karamfilov et al., 2000 & 24 & Axila & $200 \mathrm{U}$ & 5 meses & $\begin{array}{l}\text { Dor e ardência } \\
\text { locais }\end{array}$ & Não relatado \\
\hline Kinkelin et al., 2000 & 10 & Fronte & $86 \mathrm{MU}$ & 5 meses & $\begin{array}{c}\text { Fraqueza no } \\
\text { músculo da testa }\end{array}$ & $69,1 \%$ \\
\hline Heckmann et al., 1999 & 12 & Axila & $250 \mathrm{U}$ & 10 meses & Não houve & $63,7 \%$ \\
\hline Shelley et al., 1998 & 4 & Palma & $100 \mathrm{MU}$ & 7 meses & $\begin{array}{c}\text { Fraqueza } \\
\text { no músculo } \\
\text { do polegar }\end{array}$ & Não relatado \\
\hline Solomon e Hayman, 2000 & 20 & Palma & $165 \mathrm{U}$ & 7 meses & $\begin{array}{c}\text { Dor local } \\
\text { e fraqueza no } \\
\text { músculo } \\
\text { do polegar }\end{array}$ & $69 \%$ \\
\hline
\end{tabular}


durado nove meses em três pacientes. A sudorese retornou entre três e seis meses em outros dois pacientes $^{3}$.

Shelley et al. ${ }^{9}$ trataram quatro pacientes com hiperidrose palmar aplicando doses de $100 \mathrm{MU}$ de toxina em cada mão. A anidrose surgiu após uma semana da aplicação. Esse efeito durou dez meses em um paciente, sete meses em dois pacientes e quatro meses em um outro paciente. Apenas um dos pacientes relatou fraqueza transitória muscular do polegar ${ }^{9}$. No estudo de Solomon e Hayman ${ }^{10} 20$ pacientes com hiperidrose palmar e digital foram tratados com $165 \mathrm{U}$ de toxina em cada mão. Um paciente desistiu do tratamento. A anidrose ocorreu em 15 pacientes dentro de uma semana e nos quatro restantes em duas semanas. A duração da anidrose foi de nove meses em três pacientes, oito meses em três pacientes, sete meses em oito pacientes, seis meses em três pacientes, cinco meses em um paciente e quatro meses em um paciente. A redução percentual da sudorese foi de $90 \%$ em quatro pacientes, $75 \%$ em oito pacientes e $50 \%$ em sete pacientes. Todos os participantes sentiram dor durante a aplicação. Apenas quatro pacientes relataram fraqueza muscular na porção curta do músculo abdutor ${ }^{10}$.

\section{Discussão}

A toxina botulínica tipo A mostrou-se eficaz no tratamento da hiperidrose ao reduzi-la significativamente. Seus efeitos colaterais foram pouco relevantes e passageiros, não representando, assim, uma restrição ao seu uso. É um procedimento que não apresenta riscos, não há necessidade de internamento nem de anestesia de grande porte, de modo que o paciente pode retornar às suas atividades no mesmo dia. $\mathrm{O}$ aspecto que realmente limita o uso dessa toxina é o seu alto custo. Um frasco contendo 100 unidades da toxina do laboratório Allergan $\left(\right.$ Botox $^{\circledR}$ ) custa em média R\$600,00. Acrescente-se ainda o valor da sua aplicação, que no mercado atual está em torno de R $\$ 1.000,00$.

Por ser um tema atual, poucos são os artigos disponíveis na literatura. A maior parte dos artigos revisados conta com um número pequeno de pacientes, tratando-se de ensaios clínicos abertos e não controlados. As amostras foram selecionadas por conveniência (exceto Heckmann et al., 2001), tendo em vista o objetivo dos trabalhos de observar a eficácia desse tratamento em pessoas que comprovadamente tinham hiperidrose. Apesar desse tipo de amostragem e do número reduzido de casos, isso não invalida o valor desses estudos. Devido à redução expressiva da sudorese nos pacientes estudados, considera-se que esse medicamento de fato se mostrou útil para o tratamento da hiperidrose. Estudos controlados devem ser feitos para comprovação científica do medicamento; os estudos revisados, no entanto, sugerem que a toxina botulínica é um medicamento útil para o controle da hiperidrose, apesar de não se mostrar como opção definitiva.

\section{SUMMARY}

\section{Efficacy of botulinum toxin type $A$ in hyperhidrosis}

Hyperhidrosis is known as an excessive and uncontrollable sweating induced by sympathetic hyperactivity. This disease may cause social, psychological and occupational problems. Conventional treatments that uses iontoforesis, sympathectomy, antiperspirants are not effective on several cases or risky. Botulinum toxin type A appeared as a secure and efficient treatment against hyperhidrosis. It blocks acetylcholine release from presynaptic membranes. This article reviews critically the literature about this topic. The results come from six trials made with patients that had hyperhidrosis. The percentile of sweating reduction was $86 \%$ in the study made by Heckmann et al. (2001), 69,1\% in Kinkelin's et al. study, 63,7\% in Heckmann's et al. study (1999), and 69\% in the study made by Solomon et $a l$. Few side effects were related, they are pain during application, weakness on thumb and forehead muscles. Treatment of palmoplantar, axillary and frontal hyperhidrosis with botulinum toxin has been safe, effective, and patients return to normal activity the same day. The treatment is very expensive, limiting its use.

\section{Keyw ords}

Hyperhidrosis, treatment, botulinum toxin.

\section{Referências}

1. http://hiperidrose.com

2. Lewis PS. Treatment of hyperhidrosis. Dermatologic Clinics, 16(4):863-7, 1998.

3. Heckmann M, Breit S, Ceballos-Baumann A, Schaller M, Plewing $G$. Side-controlled intradermal infection of botulinum toxin $A$ in recalcitrant axillary hiperhidrosis. $J$ Am Academy Dermatol, 41(6):987-90, 1999.

4. http://www.wplastia.com.br/botox

5. Huang W, Foster J, Rogachefsky A. Pharmacology of botulinum toxin. J Am Academy Dermat, 43(2):249-59, 2000. 
6. Heckmann M, Ceballos-Baumann A, Plewig G. Botulinum toxin A for axillary hyperhidrosis (excessive sweating). New Eng J Med, 344(7):488-93, 2001.

7. Karamfilov T, Konrad H, Karte K, Wollina U. Lower relapse rate botulinum toxin $\mathrm{A}$ therapy for axillary hyperhidrosis by dose increase. Arch Dermatol, 163:487-90, 2000.

8. Kinkelin I, Hund M, Naumann M, Hamm H. Effective treatment of frontal hyperhidrosis with botulinum toxin $\mathrm{A}$. Br J Dermatol, 143(4):824-7, 2000.

9. Shelley W, Talanin N, Shelley E. Botulinum toxin therapy for palmar hyperhidrosis. J Am Academy Dermatol, 38(2):227-9, 1998.
10. Solomon B, Hayman R. Botulinum toxin Type a therapy for palmar and digital hyperhidrosis. J Am Academy Dermatol, 42 (6):1026-9, 2000.

\section{Endereço para correspondência:}

Escola Baiana de Medicina e Saúde Pública Disciplina de Farmacologia

Rua Frei Henrique, 8

CEP 40050-420 - Salvador, BA

E-mail: lisdias@ig.com.br 